\title{
A STEADY EULER FLOW WITH COMPACT SUPPORT
}

\author{
A. V. GAVRILOV
}

\begin{abstract}
A nontrivial smooth steady incompressible Euler flow in three dimensions with compact support is constructed. Another uncommon property of this solution is the dependence between the Bernoulli function and the pressure.
\end{abstract}

\section{INTRODUCTION}

A steady flow of an ideal fluid in $\mathbb{R}^{3}$ is a solution of the Euler equation

$$
(u \cdot \nabla) u=-\nabla p, \operatorname{div} u=0 .
$$

At present, it is not known if smooth nonzero solutions of this equation with compact support $0 \neq u \in C_{0}^{\infty}\left(\mathbb{R}^{3}\right)$ exist $[8]$. The problem is trivial in two dimensions where there are obvious vortex-like solutions. In three dimensions, only a few results are known, all on the negative side. It is known that such a flow cannot be Beltrami [8] 3], and it cannot be axisymmetric without swirl [6. Recently, Nadirashvili and Vladut found some other restrictions 9 .

Apparently 1 , weak solutions with compact support may be constructed using methods of [4. Also, there is a considerable literature about vortex rings which are solutions with compactly supported vorticity (e.g. [2]). Opinions have been expressed that in three dimensions there are no smooth solutions with compact support besides $u=0$. The main goal of this paper is to show that it is not true.

Theorem There exists a nontrivial smooth steady Euler flow in $\mathbb{R}^{3}$ with support in an arbitrarily small neighbourhood of a circle.

We give below an explicit description of an axisymmetric flow with compact support. This solution has also other unusual properties discussed in the last section.

Acknowledgement. The author would like to thank the anonymous referee for pointing out the interesting work of Khesin, Kuksin, and Peralta-Salas [7] as well as some properties of the given solution.

\section{Some DiffEREnTIAL EQUATIONS}

In this section we find solutions of some differential equations which will be used later.

\subsection{Ordinary differential equation.}

\section{Lemma 1}

\footnotetext{
${ }^{1}$ The author is no expert in this area.
} 
The singular Cauchy problem

$$
3 x \psi^{\prime \prime}+6 x\left(\psi^{\prime}\right)^{3}-4 \psi\left(\psi^{\prime}\right)^{2}-3 \psi^{\prime}=0 ; \psi(0)=1, \psi^{\prime}(0)=-\frac{3}{4}
$$

has a unique analytic solution $\psi(x)$ in a neighbourhood of $x=0$.

Proof. The equation (11) becomes first order in variables $t=x \psi^{-2}$ and $v=\psi \psi^{\prime}$,

$$
t \frac{d v}{d t}=v\left(\frac{4}{3} v+1\right)+\frac{t v^{2}(2 v+9)}{3(1-2 t v)} .
$$

Denoting $w=v+\frac{3}{4}$ we may write this equation as $t \frac{d w}{d t}=-w+f(t, w)$ where the function $f$ is analytic and $f(0,0)=\frac{\partial}{\partial w} f(0,0)=0$. By [5, Theorem 11.1] there is an unique analytic solution $v(t)$ such that $v(0)=-\frac{3}{4}$. The Cauchy problem $\frac{d \psi}{d x}=\frac{1}{\psi} v\left(\frac{x}{\psi^{2}}\right), \psi(0)=1$ clearly has an unique solution.

From now $\psi$ always means the function defined by (11). The Taylor series of this function is

$$
\psi(x)=1-\frac{3}{4} x+\frac{9}{128} x^{2}-\frac{21}{1024} x^{3}+\frac{1035}{131072} x^{4}-\frac{1809}{524288} x^{5}+O\left(x^{6}\right) .
$$

Denote

$$
\begin{gathered}
F(x, \alpha)=-2 x \psi(\alpha)+2 x^{3}, H(\alpha)=6 \alpha\left(\frac{1}{\psi^{\prime}(\alpha)}+2 \psi(\alpha)\right), \\
G(x, \alpha)=12 x^{2} \alpha-F^{2}(x, \alpha)-H(\alpha) .
\end{gathered}
$$

Note that at $(x, \alpha)=(1,0)$ we have $F=G=0$ and

$$
\frac{\partial F}{\partial x}=4, \frac{\partial F}{\partial \alpha}=\frac{3}{2}, \frac{\partial G}{\partial x}=0, \frac{\partial G}{\partial \alpha}=8 .
$$

We will also need the following fact

Lemma 2 The functions $F, G$ satisfy

$$
\begin{gathered}
\frac{\partial G}{\partial x}+F \frac{\partial G}{\partial \alpha}=2 G \frac{\partial F}{\partial \alpha}, \\
x \frac{\partial F}{\partial x}-F=4 x^{3} .
\end{gathered}
$$

Proof. The part (3) is trivial; (2) boils down to the formula

$$
H^{\prime}(\alpha)=24 \alpha \psi^{\prime}(\alpha)+4 \psi(\alpha)
$$

equivalent to (1). 


\subsection{Partial differential equations.}

Lemma 3 The system 2

$$
\frac{\partial}{\partial x} \alpha=F(x, \alpha),\left(\frac{\partial}{\partial y} \alpha\right)^{2}=G(x, \alpha),
$$

has a unique analytic solution $\alpha(x, y)$ in a neighbourhood of the point $(x, y)=$ $(1,0)$ such that $\alpha(1,0)=0$ and $\frac{\partial}{\partial y} \alpha \not \equiv 0$.

Proof. It is convenient to introduce an $a d$ hoc variable $s$ and to consider $x$ and $\alpha$ functions of $(F, s)$, where $G=s^{2}$ by definition. (This is possible because $\frac{\partial(F, G)}{\partial(x, \alpha)}(1,0)=32 \neq 0$.) Consider a differential form

$$
\kappa=\frac{\partial x}{\partial s} d F+\left(\frac{\partial \alpha}{\partial s}-F \frac{\partial x}{\partial s}\right) \frac{d s}{s} .
$$

It is analytic near the origin of the $(F, s)$ plane (because $s^{-1} \frac{\partial}{\partial s}=2 \frac{\partial}{\partial G}$ ). We have the relation (2) which in the new variables takes the form

$$
F \frac{\partial x}{\partial F}+s \frac{\partial x}{\partial s}=\frac{\partial \alpha}{\partial F} \text {. }
$$

It follows that

$$
d \kappa=\frac{1}{s} \frac{\partial}{\partial s}\left(F \frac{\partial x}{\partial F}+s \frac{\partial x}{\partial s}-\frac{\partial \alpha}{\partial F}\right) d s \wedge d F=0 .
$$

By the Poincare lemma, there is a unique analytic function $\Phi(F, s)$ such that $\Phi(0,0)=0$ and $\kappa=d \Phi$.

This form is odd with respect to the second variable, in the sense that $\sigma^{*} \kappa=-\kappa$ where $\sigma:(F, s) \mapsto(F,-s)$. If $\gamma$ is a path connecting the origin $(0,0)$ to a given point $(F, s)$, then

$$
\Phi(F,-s)=\int_{\sigma \gamma} \kappa=\int_{\gamma} \sigma^{*} \kappa=-\int_{\gamma} \kappa=-\Phi(F, s) .
$$

We have $\Phi^{2}(F, s)=\Phi^{2}(F,-s)$, hence the square $\Phi^{2}$ is a well defined analytic function of $F$ and $G=s^{2}$. Now we can change the variables back and denote $f(x, \alpha)=\Phi^{2}$. We have $f(1,0)=0$ (essentially, by assumptions).

Near the origin $\Phi(F, s)=\left(\frac{1}{4}+O(F)\right) s+O\left(s^{3}\right)$, hence

$$
\frac{\partial \Phi^{2}}{\partial F}(0,0)=0, \frac{\partial \Phi^{2}}{\partial G}(0,0)=\frac{1}{16},
$$

and

$$
\frac{\partial}{\partial \alpha} f(1,0)=\left(\frac{3}{2} \frac{\partial}{\partial F}+8 \frac{\partial}{\partial G}\right) \Phi^{2}(0,0)=\frac{1}{2} .
$$

By the implicit function theorem, in a neighbourhood of the origin there is a unique analytic function of two variables $\alpha(x, y)$ such that $\alpha(1,0)=0$ and

$$
f(x, \alpha(x, y))=y^{2} .
$$

\footnotetext{
${ }^{2}$ Note that $\frac{\partial}{\partial x}$ means $\left.\frac{\partial}{\partial x}\right|_{\alpha}$ when applied to $F$ or $G$ but $\left.\frac{\partial}{\partial x}\right|_{y}$ when applied to $\alpha$. To avoid a (very common) inconsistency in notation we write this partial derivative as $\frac{\partial f}{\partial x}$ for the former and $\frac{\partial}{\partial x} f$ for the latter.
} 
From (2) and the definition of $\kappa$ we have

$$
s d \Phi=d \alpha-F d x .
$$

Now, in the variables $(x, y)$ we have $\Phi^{2}=y^{2}$, hence $d \Phi^{2}=d y^{2}$ and 3

$$
(d \alpha-F(x, \alpha) d x)^{2}-G(x, \alpha) d y^{2}=0 .
$$

This equality implies (4) (and is essentially equivalent to it).

Remark 1 In Lemma 3, the condition $\alpha(1,0)=0$ is crucial. In this case we cannot take the square root of the second equation, and solving the system is more difficult then for $\alpha(1,0)>0$. Unfortunately, in the latter case the function $\alpha$ would have no extrema, and the Euler flow $u$ in the following section could not be extended to the whole space.

Remark 2 Note that $\alpha(x, y)=\alpha(x,-y)$ pretty much by definition. An interesting consequence is $G(x, \alpha(x, 0))=0$. (Which follows from (4) and $\frac{\partial}{\partial y} \alpha(x, 0)=0$.) Because of this, the function $\alpha(x, 0)$ is actually another analytic solution of (4).

Remark 3 In the given proof, the main technical difficulty is the absence of an inverse map to $(F, s) \mapsto(x, \alpha)$. We circumvent this obstacle by artificially constructing a function $\Phi^{2}(F, s)$ with a well defined "pullback". One of the referees pointed out that there is a more straightforward (although not unrelated) proof using the Cartan - Kähler Theorem. We may consider a form $\omega=d \alpha-p d x-q d y$ on a manifold of dimension 3 defined by equations

$$
p=F(x, \alpha), q^{2}=G(x, \alpha)
$$

in variables $x, y, \alpha, p, q$. This form satisfies the integrability condition $\omega \wedge d \omega=0$, so we can use it to construct the function $\alpha(x, y)$. (An important detail is that $\omega / q$ is analytic.)

Remark 4 The method of the proof is constructive and may be used to compute the Taylor series

$$
\alpha(x, y)=2(x-1)^{2}+2 y^{2}+3(x-1)^{3}+3(x-1) y^{2}+O\left((|x-1|+|y|)^{4}\right) .
$$

The first two terms are important, so it may be appropriate to include a direct computation of them.

Lemma 4 The function $\alpha(x, y)$ has a strict local minimum at $(x, y)=(1,0)$.

Proof. The first derivatives at this point are zero by (44). We have

$$
\frac{\partial^{2}}{\partial x^{2}} \alpha=\frac{\partial}{\partial x} F=\frac{\partial F}{\partial x}=4, \frac{\partial^{2}}{\partial x \partial y} \alpha=\frac{\partial}{\partial y} F=\left(\frac{\partial F}{\partial \alpha}\right)\left(\frac{\partial}{\partial y} \alpha\right)=0 .
$$

Finally, (at any point) we have the equality

$$
\left(\frac{\partial G}{\partial \alpha}\right)\left(\frac{\partial}{\partial y} \alpha\right)=\frac{\partial}{\partial y} G=2\left(\frac{\partial}{\partial y} \alpha\right)\left(\frac{\partial^{2}}{\partial y^{2}} \alpha\right) .
$$

As the derivative $\frac{\partial}{\partial y} \alpha$ is not identically zero, it implies

$$
\frac{\partial^{2}}{\partial y^{2}} \alpha=\frac{1}{2} \frac{\partial G}{\partial \alpha} \text {. }
$$

\footnotetext{
${ }^{3}$ As customary, $d y^{2}$ actually means $d y \otimes d y$, a tensor square.
} 
At the point under consideration we have then $\frac{\partial^{2}}{\partial y^{2}} \alpha=4$. The second differential $d^{2} \alpha$ is positively definite, so this point is a strict minimum.

\section{THE FLOW}

We use the standard cylindrical coordinates. For a velocity field with axial symmetry the Euler equation $(u \cdot \nabla) u=-\nabla p$ takes the form

$$
\left\{\begin{array}{r}
u_{\rho} \frac{\partial}{\partial \rho} u_{\rho}+u_{z} \frac{\partial}{\partial z} u_{\rho}-\frac{1}{\rho} u_{\varphi}^{2}=-\frac{\partial}{\partial \rho} p \\
u_{\rho} \frac{\partial}{\partial \rho} u_{\varphi}+u_{z} \frac{\partial}{\partial z} u_{\varphi}+\frac{1}{\rho} u_{\rho} u_{\varphi}=0 \\
u_{\rho} \frac{\partial}{\partial \rho} u_{z}+u_{z} \frac{\partial}{\partial z} u_{z}=-\frac{\partial}{\partial z} p .
\end{array}\right.
$$

For $R>0$, denote $a=\alpha\left(\frac{\rho}{R}, \frac{z}{R}\right)$. For the sake of convenience, we denote by $\mathcal{C}$ the circle $\rho=R, z=0$ where $a=0$. Let

$$
p=\frac{a R^{4}}{4}, b=\frac{R^{3}}{4} \sqrt{H(a)}, u=\frac{1}{\rho}\left(\frac{\partial p}{\partial z} e_{\rho}-\frac{\partial p}{\partial \rho} e_{z}+b e_{\varphi}\right) .
$$

(Note that $b$ is not smooth on $\mathcal{C}$.) Obviously, $\operatorname{div} u=0$ outside $\mathcal{C}$.

The fields $(u, p)$ given by (6) satisfy (5) in a neighbourhood of $\mathcal{C}$ (but not on the curve itself).

Proof. The second equation of (5) is obvious. The last one is equivalent to

$$
x\left(\frac{\partial}{\partial x} \alpha\right) \frac{\partial^{2}}{\partial x \partial y} \alpha+\left(\frac{\partial}{\partial y} \alpha\right)\left(\frac{\partial}{\partial x} \alpha+4 x^{3}-x \frac{\partial^{2}}{\partial x^{2}} \alpha\right)=0,
$$

where $x=\frac{\rho}{R}, y=\frac{z}{R}$ and $\alpha=\alpha(x, y)$. After multiplication by $\frac{\partial}{\partial y} \alpha$, using (4) and

$$
\frac{\partial G}{\partial x}+F \frac{\partial G}{\partial \alpha}=\frac{\partial}{\partial x}\left(\frac{\partial}{\partial y} \alpha\right)^{2}=2\left(\frac{\partial}{\partial y} \alpha\right) \frac{\partial^{2}}{\partial x \partial y} \alpha
$$

we have

$$
\frac{1}{2} x F\left(\frac{\partial G}{\partial x}+F \frac{\partial G}{\partial \alpha}\right)+G\left(F+4 x^{3}-x\left(\frac{\partial F}{\partial x}+F \frac{\partial F}{\partial \alpha}\right)\right)=0,
$$

which follows from (2), (3).

Finally, the first equation is

or

$$
x\left(\frac{\partial}{\partial x} \alpha\right) \frac{\partial^{2}}{\partial y^{2}} \alpha-x\left(\frac{\partial}{\partial y} \alpha\right) \frac{\partial^{2}}{\partial x \partial y} \alpha+\left(\frac{\partial}{\partial y} \alpha\right)^{2}-4 x^{3} \frac{\partial}{\partial x} \alpha+H(\alpha)=0,
$$

$$
\frac{1}{2} x F \frac{\partial G}{\partial \alpha}-\frac{1}{2} x\left(\frac{\partial G}{\partial x}+F \frac{\partial G}{\partial \alpha}\right)+G-4 x^{3} F+H(\alpha)=0,
$$

which is again a consequence of (2), (3).

As introduced, this Euler flow is only defined in a vicinity of the circle $\mathcal{C}$. However, this flow satisfies an additional condition $u \cdot \nabla p=0$ which is very useful for our purposes. Consider another field $\widetilde{u}=\omega(p) u$ where $\omega$ is a smooth function. Due to the above condition we have

$$
\operatorname{div} \widetilde{u}=\omega(p) \operatorname{div} u+\omega^{\prime}(p)(u \cdot \nabla p)=0
$$

and

$$
(\widetilde{u} \cdot \nabla) \widetilde{u}=\omega^{2}(p)(u \cdot \nabla) u+\omega(p) \omega^{\prime}(p)(u \cdot \nabla p) u=-\omega^{2}(p) \nabla p .
$$


So, regardless of a choice of the function $\omega$, the field $\widetilde{u}$ is also an Euler flow, with the corresponding pressure determined by $d \widetilde{p}=\omega^{2}(p) d p$.

Due to Lemma 4, we may assume that $\omega=\omega(p)$ in a vicinity of the circle $\mathcal{C}$ and $\omega=0$ outside this domain. If $\operatorname{supp}(\omega) \subset[\varepsilon, 2 \varepsilon]$ (as a function of $p$ ) with $\varepsilon>0$ sufficiently small, then we have $\widetilde{u} \in C^{\infty}\left(\mathbb{R}^{3}\right)$. The new flow is supported in a toroidal domain which can be made arbitrarily close to the circle. This completes the proof of Theorem 1 .

Remark 5 The poloidal stream function $\Psi=a$ is a solution of the GradShafranov equation in the following form $(R=1)$

$$
\left(\partial_{\rho \rho}+\partial_{z z}-\frac{1}{\rho} \partial_{\rho}\right) \Psi=10 \rho^{2}-\frac{1}{2} H^{\prime}(\Psi) .
$$

\section{Generalized Beltrami flows}

The condition $u \cdot \nabla p=0$ mentioned above means that the pressure is constant along a streamline. This is very uncommon, and the only other nontrivial example known to the author is a flow on the 3-sphere constructed in [7. (The trivial examples are vortices (rotational flows) and their variations.) By the Bernoulli theorem $|u|^{2}$ must also be a first integral; indeed, from (6), (4) and the definition of $G$ we have

$$
|u|^{2}=\frac{1}{\rho^{2}}\left[\left(\frac{\partial p}{\partial z}\right)^{2}+\left(\frac{\partial p}{\partial \rho}\right)^{2}+b^{2}\right]=3 p .
$$

For the modified flow $\widetilde{u}$ the formula is different but $|\widetilde{u}|^{2}$ is still a function of the pressure $\widetilde{p}$.

Recall that for a Beltrami flow $u$ the Bernoulli function $B=p+\frac{1}{2}|u|^{2}$ is constant. The case when the Bernoulli function depends on the pressure may be considered a generalization, and constructed flows belongs to this category $\left(B=\frac{5}{2} p\right.$ for the original flow). As was pointed out by Arnold [1][II.1.B], for a non-constant $B$ both the streamlines and the vortex lines lie on the surfaces $B=$ const; in our situation these are the same as $p=$ const. It makes a difference because in this case the flow sheets become independent in a sense, so the flow may be "modulated" (a trick we used in the previous section).

One of the referees pointed out to the author that a generalized Beltrami flow (with an extremum of pressure at some point) has a peculiar restriction on the behaviour of the pressure. Let $(u, p)$ be such a flow, and assume that $|u|^{2}=3 p$ as before (we can do this without loss of generality). By the same recipe as above we may then construct another flow $(\widetilde{u}, \widetilde{p})$. If it has compact support then (e.g. $[\mathbf{3}$ )

$$
\int_{\mathbb{R}^{3}}\left(|\widetilde{u}|^{2}+3 \widetilde{p}\right) d x=0
$$

To make sense of this it is convenient to introduce a function $V(c)=\operatorname{Vol}\left(\left\{x \in \mathbb{R}^{3}\right.\right.$ : $p(x) \leq c\})$. The equality then becomes

$$
\int_{\mathbb{R}^{3}}\left(p \omega^{2}(p)+\widetilde{p}\right) d x=\int \omega^{2}(p)(p d V(p)-V(p) d p)=0 .
$$

It must be frue for any function $\omega$ which means $V(p)=p \cdot$ const.

\footnotetext{
${ }^{4}$ This is probably what an expert would expect in this situation, but the author does not know an appropriate reference to make it a meaningful discussion.
} 


\section{REFERENCES}

[1] V.I. Arnold, B.A. Khesin, Topological Methods in Hydrodynamics. Springer, 1999.

[2] A. Ambrosetti, M. Struwe. "Existence of steady vortex rings in an ideal fluid." Arch. Rat. Mech. Anal. 108 (1989), 97-109.

[3] D. Chae, P. Constantin, "Remarks on a Liouville-type theorem for Beltrami flows." Int. Math. Res. Notices 2015 (2015), 10012-10016.

[4] A. Choffrut, L. Szekelyhidi. "Weak solutions to the stationary incompressible Euler equations". SIAM J. Math. Anal. 46 (2014), 4060-4074.

[5] E. Hille, Ordinary differential Equations in the Complex Domain. Dover Publications, 1997.

[6] Q. Jiu, Z. Xin, "Smooth Approximations and Exact Solutions of the 3D Steady Axisymmetric Euler Equations." Comm. Math. Phys. 287 (2009), 323-350.

[7] B. Khesin, S. Kuksin, D. Peralta-Salas, "KAM theory and the 3D Euler equation." Advances in Math. 267 (2014) 498-522.

[8] N. Nadirashvili, "Liouville theorem for Beltrami flow." Geometric and Functional Analysis 24 (2014), 916-921.

[9] N. Nadirashvili, S. Vladut, "Integral geometry of Euler equations." Arnold Math. J. 3 (2017), 397-421.

E-mail address: gavrilov19@gmail.com 